\title{
Impact of Sheath Blight Disease of Paddy on Seedlings and Plant Vigor
}

\author{
Nitin Kumar Toorray*, P. K. Tiwari, A. S. Kotasthane and G. K. Awadhiya \\ IGKV, Raipur, Chhhattisgarh, India, India \\ *Corresponding author
}

\section{A B S T R A C T}

\section{Keywords}

Rhizoctonia solani, Seed-borne inoculums, Effect on seeds, Seedlings and plant vigor

\section{Article Info}

Accepted:

17 September 2019

Available Online:

10 October 2019

\begin{abstract}
Rhizoctonia solani causing sheath blight of rice is reported to survive through soil and collateral hosts. Although it infects seed in field, role of seed-borne inoculum in disease development is not properly understood. However, only little information is available on the role of seed-borne inoculum in disease development in the field (Roy, 1989; Acharya and Gupta, 1996; Silvalingam et al., 2006). During the present investigation seed samples of two varieties were collected from healthy seeds (i.e. seeds harvested from healthy plant panicles) and diseased seeds (i.e. seeds harvested from plant panicles with 7 to 9 disease score of sheath blight) and were sown both under green house (pots) and field conditions. The seedling growth assessed in terms of seedling vigour index at 21 days and 30 days after sowing. The germination percent, root and shoot length of individual seedlings also be measured. The results showed that the seedling vigor index was more in healthy plant seeds than the diseased plant seeds at both observation periods. They were also statistically significant. Highest percent germination of two varieties (81.75 and 88.75$)$ were recorded when the seeds from healthy plants panicle. The root and shoot length were also affected in diseased plant seeds during the same period of observation. The seedling vigour index calculated clearly revealed that healthy plant seeds in both the varieties i.e., Swarna (2595.07) and Mahamaya (3681.14) was more than the corresponding diseased plant seeds (i.e., 1837.14 and 2762.62, respectively). The reduced growth of root, shoot and seedling vigour from diseased seeds showed that due to the survival of pathogen in diseased plant seeds and its transmission from seed to emerging seedlings, the Rhizoctonia solani pathogen is internally seed-borne in nature.
\end{abstract}

\section{Introduction}

Seed is the most elementary and specific resource of agriculture and also the basic of agriculture production. To improve the quality of the seed is one of the important measure of ensuring the high efficiency, quality and productivity. Seed vigor is the important index of measuring the seed quality. Seed vigor tests are used by researchers to predict seedling emergence under field conditions. A seed vigor test can reflect the potential of seed in the field or under storage (Zhang et al., 2007). Seed-borne disease causes enormous losses to our crop. The infected seeds may sometimes fail to germinate, transmit disease from seed to 
seedling and from seedling to 26 growing plants (Fakir et al., 2002). Seed vigour was recognized by earlier researchers as an important quality parameter and distinct from germinability. Decline in germinability was also attributed to decline in vigour and might be due to several factors including the seed infection with micro-organisms (Seshu et al., 1987). Anselme (1987) suggested that the evaluation of germination capacity, vigour and dormancy were also an important parameter in addition to the studies about infection by bacteria, fungi and viruses etc. Abnormal and diseased seeds in a seed lot also indicate poor seed health. Seed health primarily refers to the presence or absence of disease causing organisms of various kinds (International Seed Testing Association (ISTA), 1985). Ou, 1985 and Neergaard, 1979 observed that among 55 fungi that reported to be pathogenic in rice 43 are seed borne or seed transmissible. The most frequently isolated fungi from rice seed ranged from minor pathogens to major pathogens of economic importance to the rice industry (Reddy and Khare, 1977; Zainum and Nik, 1977) Kota et al., (1987) reported that the fungus causing blast, brown spot, bakanae, urbatta and stack burn are internally seed borne and result in diseased seedlings.

Sheath blight (caused by Rhizoctonia solani) which was once a minor disease, has of-late become a major disease inflicting heavy losses, and is considered to be an important disease next to rice blast in most of the Asian and also in number of states in India (Dath, 1990). Seed-borne disease causes enormous losses to our crop. Seed health primarily refers to the presence or absence of disease causing organisms of various kinds (International Seed Testing Association (ISTA), 1985). Due to the survival of pathogen in diseased plant seeds and its transmission from seed to emerging seedlings, the Rhizoctonia solani pathogen is internally seed-borne in nature (Silvalingam et al., 2006). Pathogen is reported to survive in the soil during winter as sclerotia or as mycelium. The fungus also survives on a number of collateral hosts during off-season (Kannaiyan and Prasad, 1981). However, only little information is available on the role of seed-borne inoculum in disease development in the field (Roy, 1989; Acharya and Gupta, 1996).

\section{Materials and Methods}

This trial was conducted both under green house (pots) and field conditions. There were 2 varieties (Swarna and Mahamaya), 4 treatments in a factorial RBD with 4 replications under Pot conditions and 4 replications under field conditions.

The treatments were healthy seeds (i.e., seeds harvested from healthy plant panicle and diseased seed (i.e., seeds harvested from plant panicles with 7 to 9 disease score) of both the varieties. For the pot studies, 16 earthen pots of 12 inch height with 12 inch diameter were filled with $4 \mathrm{~kg}$ field soil; and 100 seeds from each seed source of test varieties (Swarna and Mahamaya) were sown in each pot. The following observations were taken:

\section{Estimation of plant vigour index}

The seedling growth was assessed in terms of seedling vigour index at 21 days and 30 days after sowing. The germination percent was first recorded for each treatment / varieties. The root and shoot length of individual seedlings were also measured. The shoot length was taken from randomly selected ten plants and measured from the base of the shoot to upper most leaf tip. For measuring the length of the root first plant were carefully uprooted (when seedlings were 21 and 30 days old, respectively), gently washed, fully stretched and carefully placed on clean transparent surface. The length of root system was measured form up to the longest tip. 
The seedling vigour index was calculated by using the formula described by Abdul Baki and Anderson (1973).

Seedling vigour index $=($ mean root length + mean shoot length) $\mathrm{X}$ germination percent

Percent increase or decrease in growth parameters and seedling vigor index in diseases seeds (T1) over healthy seeds (T2) were calculated using formula given as:

Percent increase or decrease (in disease severity / growth parameters / yield parameters etc.) over control was calculated using the following formula in all cases where needed:

I or $\mathrm{D}=\mathrm{T}-\mathrm{C} / \mathrm{C} \times 100$

Where, I or $\mathrm{D}=$ percent increase or decrease over control

$\mathrm{C}=$ percent in control

$\mathrm{T}=$ percent in treatment

\section{Results and Discussion}

\section{Pot condition}

During the year 2016 the data in table 1 shows that In both Swarna and Mahamaya the highest percent germination were obtained when the seeds from healthy plants panicle (T1) (77.0 and 80.5, respectively). The seeds harvested from diseased plant panicle (T2) recorded 63.5 (Swarna) and 66.75 (Mahamaya). The seeds from diseased panicles showed reduction in percent germination for Swarna (63.5) or Mahamaya (66.75). The shoot length was also more in healthy plants seeds than diseased for both the varieties Swarna and Mahamaya (15.62, 18.11 in healthy 13.09 and 14.43 in diseased plant seeds, respectively. The seedling vigor index during the same period they were statistically significance between healthy and diseased plant seeds in both varieties after 21 days of sowings. The seedling vigour index calculated clearly revealed that healthy plant seeds in both the varieties i.e., Swarna (1590.82) and Mahamaya (1936.83) was more than the corresponding diseased plant seeds (i.e., 1129.92 and 1334.40, respectively). Similar trend was observed in root length of the healthy plant seed of Swarna (8.13) and Mahamaya (10.88) after 30 DAS. The diseased plant seed during the same period recorded root length of 6.77 and 8.62, respectively. The shoot length for the healthy plant seed for Swarna was 19.46 and 24.81 for Mahamaya. The shoot length was also affected in diseased plants seeds which were 16.70 and 20.23 , respectively during the same period of observation. The seedling vigour index calculated clearly revealed that healthy plant seeds in both the varieties i.e., Swarna (2129.05) and Mahamaya (2870.83) was more than the corresponding diseased plant seeds (i.e., 1489.71 and 1921.89, respectively). The statistical analysis also clearly revealed that root, shoot and seedling vigor index differences were significant between the healthy plant seeds (T1) to that of diseased plant seeds (T2) (Table 2-4).

During the year 2017 the data in table 5 shows that 21 days after sown seedlings in both Swarna and Mahamaya the highest percent germination were obtained when the seeds from healthy plants panicle (T1) (75.0 and 78.0, respectively). The seeds harvested from diseased plant panicle (T2) recorded 63.5 (Swarna) and 66.75 (Mahamaya). The seeds from diseased panicles showed reduction in percent germination for Swarna (60.5) or Mahamaya (65.25). The seeds from diseased panicles showed reduction in percent germination for Swarna (60.5) or Mahamaya (65.25). The shoot length was also more in healthy plants seeds than diseased for both the 
varieties Swarna and Mahamaya (15.03, 18.09 in healthy 13.38 and 14.53 in diseased plant seeds, respectively. The seedling vigor index during the same period they were statistically significance between healthy and diseased plant seeds in both varieties after 21 days of sowings. On 21 days after sown seedlings the seedling vigour index calculated clearly revealed that healthy plant seeds in both the varieties i.e., Swarna (1520.68) and Mahamaya (1897.68) was more than the corresponding diseased plant seeds (i.e., 1097.79 and 1347.23, respectively). On 30 days after sown seedlings the seedling vigour index calculated clearly revealed that healthy plant seeds in both the varieties i.e., Swarna (2087.26) and Mahamaya (2761.67) was more than the corresponding diseased plant seeds (i.e., 1403.64 and 1876.97, respectively). Similar trend was observed in root length of the healthy plant seed of Swarna (8.09) and Mahamaya (10.77) after 30 DAS. The diseased plant seed during the same period recorded root length of 6.76 and 8.57, respectively. The shoot length for the healthy plant seed for Swarna was 19.70 and 24.66 for Mahamaya. The shoot length was also affected in diseased plants seeds which were 16.44 and 20.17 , respectively during the same period of observation. The seedling vigour index calculated clearly revealed that healthy plant seeds in both the varieties i.e., Swarna (2087.26) and Mahamaya (2761.67) was more than the corresponding diseased plant seeds (i.e., 1403.64 and 1876.97 , respectively). The statistical analysis also clearly revealed that root, shoot and seedling vigor index differences were significant between the healthy plant seeds (T1) to that of diseased plant seeds (T2).

The pooled data clearly shows in table 9 and 10 that in both Swarna and Mahamaya the highest percent germination were obtained when the seeds from healthy plants panicle (T1) (76.0 and 79.25, respectively).
The seeds harvested from diseased plant panicle (T2) recorded 62.0 (Swarna) and 66.0 (Mahamaya). On 21 days after sown seedlings the shoot length was also more in healthy plants seeds than diseased for both the varieties Swarna and Mahamaya (15.32, 18.10 in healthy 13.23 and 14.48 in diseased plant seeds, respectively. On 30 days after sown seedlings the shoot length was also more in healthy plants seeds than diseased for both the varieties Swarna and Mahamaya (19.58, 24.73 in healthy 16.57 and 20.20 in diseased plant seeds, respectively. The seedling vigor index during the same period they were statistically significance between healthy and diseased plant seeds in both varieties after 21 days of sowings. The seedling vigour index calculated clearly revealed that healthy plant seeds in both the varieties i.e., Swarna (1555.75) and Mahamaya (1917.25) was more than the corresponding diseased plant seeds (i.e., 1113.85 and 1340.81 , respectively).

Similar trend was observed in root length of the healthy plant seed of Swarna (8.11) and Mahamaya (10.82) after 30 DAS. The diseased plant seed during the same period recorded root length of 6.76 and 8.59, respectively. The shoot length for the healthy plant seed for Swarna was 19.58 and 24.73 for Mahamaya.

The shoot length was also affected in diseased plants seeds which were 16.57 and 20.20, respectively during the same period of observation. The seedling vigour index calculated clearly revealed that healthy plant seeds in both the varieties i.e., Swarna (2108.15) and Mahamaya (2816.25) was more than the corresponding diseased plant seeds (i.e., 1446.67 and 1899.43, respectively). The statistical analysis also clearly revealed that root, shoot and seedling vigor index differences were significant between the healthy plant seeds (T1) to that of diseased plant seeds (T2). 


\section{Field condition}

During the year 2016 the data in table 3 and 4 shows that under field conditions also similar trends was noticed in both varieties at 21 days and 30 days after sowing (Table 3 and 4). The root length for Swarna healthy plant panicle seeds were $5.95 \mathrm{~cm}$ compared to the same variety seeds collected from disease plant panicle i.e., $8.73 \mathrm{~cm}$ Similarly the shoot length were $18.04 \mathrm{~cm}$ and $25.65 \mathrm{~cm}$ for Swarna and $23.13 \mathrm{~cm}$ and $19.70 \mathrm{~cm}$ for Mahamaya, at 21 days respectively for the healthy and diseased seed tested and $33.27 \mathrm{~cm}$ and $31.56 \mathrm{~cm}$ for Mahamaya at 30 days respectively for the healthy and diseased seeds tested. The seedling vigor index was more in healthy plant seeds (1913.98 in Swarna and 2551.59 in Mahamaya in 21 days for healthy seeds and 2747.24 in swarna and 3714.33 in mahamaya in 30 days for healthy seeds) than the diseased plant seed at both observation periods. They were also statistically significant. Similar findings were also reported Fakir et al., (2002) who stated that the infected seeds may sometimes fail to germinate, transmit disease from seed to 52 seedling and from seedling to growing plants. Nanda and Chaudhary (1972) carried out studies in tropical Asia and reported that germination percent was higher (98 percent), as well as faster for normal than for diseased / spotted seeds ( $<90$ percent). Ou, 1985 and Neergaard, 1979 observed that among 55 fungi that reported to be pathogenic in rice 43 are seed borne or seed transmissible. Binesh and Torabi (1985) reported seed samples collected from infected rice fields and tested When infected seeds were sown in sterile soil up to 5 percent of the seedlings were killed by $R$. solani and 6.2-11.7 percent of the surviving plants developed sheath blight symptoms.

During the year 2017 the data in table 7 and 8 shows that under field conditions also similar trends was noticed in both varieties at 21 days and 30 days after sowing. The root length for Swarna healthy plant panicle seeds were 6.12 $\mathrm{cm}$ compared to the same variety seeds collected from disease plant panicle i.e., 5.35 $\mathrm{cm}$ Similarly the shoot length were $18.40 \mathrm{~cm}$ and $16.24 \mathrm{~cm}$ for Swarna and $23.71 \mathrm{~cm}$ and $19.59 \mathrm{~cm}$ for Mahamaya, at 21 days respectively for the healthy and diseased seed tested and $33.47 \mathrm{~cm}$ and $31.16 \mathrm{~cm}$ for Mahamaya at 30 days respectively for the healthy and diseased seeds tested. The seedling vigor index was more in healthy plant seeds (1920.22 in Swarna and 2548.08 in Mahamaya in 21 days for healthy seeds and 2442.93 in swarna and 3647.95 in mahamaya in 30 days for healthy seeds) than the diseased plant seed at both observation periods. They were also statistically significant.

The percent germination, root length, shoot length and seedling vigor index were significantly higher in both varieties (Swarna and Mahamaya) in the healthy plant seeds (T1) as compared to the diseased plant seeds (T2), in both trials conducted under the green house (pot) and field conditions and also at both observation (21 and 30 DAS).

The pooled data clearly shows in table 11 and 12 that In both Swarna and Mahamaya the highest percent germination were obtained when the seeds from healthy plants panicle (T1) (79.0 and 81.12, respectively). The seeds harvested from diseased plant panicle (T2) recorded 64.62 (Swarna) and 67.0 (Mahamaya). On 21 days after sown seedlings the shoot length was also more in healthy plants seeds than diseased for both the varieties Swarna and Mahamaya (18.22, 23.42 in healthy and 16.29, 19.64 in diseased plant seeds, respectively. On 30 days after sown seedlings the shoot length was also more in healthy plants seeds than diseased for both the varieties Swarna and Mahamaya (25.76, 33.37 in healthy and 20.83 and 31.36 in diseased plant seeds, respectively (Fig. 1). 
Table.1 Effect of sheath blight incidence on seedling vigor under Pot conditions (Year-2016)

\begin{tabular}{|c|c|c|c|c|c|c|c|c|c|c|c|c|c|c|}
\hline \multirow{4}{*}{$\begin{array}{c}\begin{array}{c}\text { Trea } \\
\text { tme } \\
\text { nt }\end{array} \\
\text { T1 }\end{array}$} & \multirow{2}{*}{\multicolumn{2}{|c|}{$\begin{array}{c}\text { Seed Source } \\
\text { (Quality) }\end{array}$}} & \multirow{2}{*}{\multicolumn{2}{|c|}{$\%$ Germination }} & \multirow[t]{3}{*}{ Avg. } & \multicolumn{9}{|c|}{21 Days After Sown Seedlings } \\
\hline & & & & & & \multicolumn{2}{|c|}{$\begin{array}{c}\text { Root } \\
\text { length }(\mathrm{cm})\end{array}$} & \multirow[t]{2}{*}{ Avg. } & \multicolumn{2}{|c|}{$\begin{array}{l}\text { Shoot length } \\
(\mathrm{cm})\end{array}$} & \multirow[t]{2}{*}{ Avg. } & \multicolumn{2}{|c|}{$\begin{array}{l}\text { Seedling vigor } \\
\text { index }\end{array}$} & \multirow[t]{2}{*}{ Avg. } \\
\hline & \multirow{2}{*}{\multicolumn{2}{|c|}{$\begin{array}{l}\text { Seeds from } \\
\text { healthy plant } \\
\text { panicle }\end{array}$}} & V1 & $\mathrm{V} 2$ & & V1 & $\mathrm{V} 2$ & & V1 & $\mathrm{V} 2$ & & V1 & V2 & \\
\hline & & & 77.0 & 80.5 & 78.75 & 5.04 & 5.95 & 5.49 & 15.62 & 18.11 & 16.86 & 1590.82 & 1936.83 & 1763.82 \\
\hline $\mathbf{T 2}$ & \multicolumn{2}{|c|}{$\begin{array}{c}\text { Seeds from } \\
\text { diseased plant } \\
\text { panicle }\end{array}$} & 63.5 & 66.75 & 65.12 & 4.67 & 5.5 & 5.08 & 13.09 & 14.43 & 13.76 & 1129.92 & 1334.40 & 1232.16 \\
\hline \multicolumn{3}{|c|}{ Average } & 70.25 & 73.62 & 71.93 & 4.85 & 5.72 & 5.28 & 14.35 & 16.27 & 15.31 & 1360.37 & 1635.61 & 1497.99 \\
\hline \multirow{3}{*}{\multicolumn{2}{|c|}{$\mathrm{SE}(\mathbf{m})+$}} & \multicolumn{3}{|c|}{ Variety (V) } & 1.80 & & & 0.23 & & & 0.43 & & & 48.68 \\
\hline & & \multicolumn{3}{|c|}{ Seed Source (Quality)(T) } & 1.80 & & & 0.23 & & & 0.43 & & & 48.68 \\
\hline & & \multicolumn{3}{|c|}{ V x T } & 2.54 & & & 0.32 & & & 0.61 & & & 68.84 \\
\hline \multirow{3}{*}{\multicolumn{2}{|c|}{ CD (5\%) }} & \multicolumn{3}{|c|}{ Variety $(\mathrm{V})$} & 5.76 & & & 0.72 & & & 1.38 & & & 155.73 \\
\hline & & \multicolumn{3}{|c|}{ Seed Source (Quality)(T) } & 5.76 & & & 0.72 & & & 1.38 & & & 155.73 \\
\hline & & \multicolumn{3}{|c|}{$\mathrm{V} \times \mathrm{T}$} & 8.14 & & & 1.02 & & & 1.95 & & & 220.23 \\
\hline \multicolumn{2}{|c|}{ CV (\%) } & & & & 7.07 & & & 11.79 & & & 7.86 & & & 9.19 \\
\hline
\end{tabular}

V1- Swarna V2- Mahamaya *Average of four replications *Square root transformation

Table.2 Effect of sheath blight incidence on seedling vigor under Pot conditions (Year-2016)

Design - Factorial RBD; Treatments-4; Replication-4

\begin{tabular}{|c|c|c|c|c|c|c|c|c|c|c|c|c|c|c|}
\hline \multirow{4}{*}{$\begin{array}{c}\begin{array}{c}\text { Trea } \\
\text { tme } \\
\text { nt }\end{array} \\
\text { T1 }\end{array}$} & \multirow{2}{*}{\multicolumn{2}{|c|}{$\begin{array}{c}\text { Seed Source } \\
\text { (Quality) }\end{array}$}} & \multirow{2}{*}{\multicolumn{2}{|c|}{$\%$ Germination }} & \multirow[t]{3}{*}{ Avg. } & \multicolumn{9}{|c|}{30 Days After Sown Seedlings } \\
\hline & & & & & & \multicolumn{2}{|c|}{$\begin{array}{c}\text { Root } \\
\text { length }(\mathrm{cm})\end{array}$} & \multirow[t]{2}{*}{ Avg. } & \multicolumn{2}{|c|}{$\begin{array}{l}\text { Shoot length } \\
(\mathrm{cm})\end{array}$} & \multirow[t]{2}{*}{ Avg. } & \multicolumn{2}{|c|}{$\begin{array}{c}\text { Seedling vigor } \\
\text { index }\end{array}$} & \multirow[t]{2}{*}{ Avg. } \\
\hline & \multirow{2}{*}{\multicolumn{2}{|c|}{$\begin{array}{l}\text { Seeds from } \\
\text { healthy plant } \\
\text { panicle }\end{array}$}} & V1 & $\mathrm{V} 2$ & & $\mathrm{~V} 1$ & $\mathrm{~V} 2$ & & V1 & $\mathrm{V} 2$ & & V1 & $\mathrm{V} 2$ & \\
\hline & & & 77.0 & 80.5 & 78.75 & 8.13 & 10.88 & 9.50 & 19.46 & 24.81 & 22.13 & 2129.05 & 2870.83 & 2499.94 \\
\hline $\mathbf{T 2}$ & \multicolumn{2}{|c|}{$\begin{array}{l}\text { Seeds from } \\
\text { diseased plant } \\
\text { panicle }\end{array}$} & 63.5 & 66.75 & 65.12 & 6.77 & 8.62 & 7.69 & 16.70 & 20.23 & 18.46 & 1489.71 & 1921.89 & 1705.8 \\
\hline \multicolumn{3}{|c|}{ Average } & 70.25 & $\mathbf{7 3 . 6 2}$ & 71.93 & 7.45 & 9.75 & 8.59 & 18.08 & 22.52 & 20.29 & 1809.38 & 2396.36 & 2102.87 \\
\hline \multirow{3}{*}{\multicolumn{2}{|c|}{$\mathbf{S E}(\mathbf{m})+$}} & & \multicolumn{2}{|c|}{ Variety $(\mathrm{V})$} & 1.80 & & 0.25 & \multicolumn{2}{|c|}{ 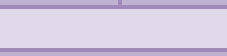 } & 0.36 & \multicolumn{2}{|c|}{ 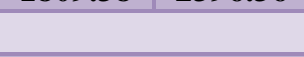 } & 95.20 \\
\hline & & \multicolumn{3}{|c|}{ Seed Source (Quality)(T) } & 1.80 & & & 0.25 & & 0.36 & & 95.20 \\
\hline & & \multicolumn{3}{|c|}{$\mathrm{V} \times \mathrm{T}$} & 2.54 & & & 0.36 & & 0.51 & \multicolumn{2}{|c|}{ 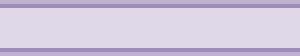 } & 134.63 \\
\hline \multirow{3}{*}{\multicolumn{2}{|c|}{ CD $(5 \%)$}} & \multicolumn{3}{|c|}{ Variety $(\mathrm{V})$} & 5.76 & & & 0.81 & & 1.16 & \multicolumn{2}{|c|}{+2} & 304.56 \\
\hline & & \multicolumn{3}{|c|}{ Seed Source (Quality)(T) } & 5.76 & & & 0.81 & & 1.16 & \multirow{2}{*}{\multicolumn{2}{|c|}{ 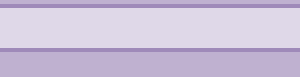 }} & 304.56 \\
\hline & & \multirow{2}{*}{\multicolumn{3}{|c|}{$\mathrm{V} \times \mathrm{T}$}} & 8.14 & & & 1.15 & & 1.64 & & & 430.71 \\
\hline \multicolumn{2}{|c|}{ CV (\%) } & & & & 7.07 & & & 8.36 & & & 5.04 & & & 13.16 \\
\hline
\end{tabular}

V1- Swarna V2- Mahamaya *Average of four replications *Square root transformation 
Table.3 Effect of sheath blight incidence on seedling vigor under field conditions (Year-2016)

Design - Factorial RBD; Treatments-4; Replication-4

\begin{tabular}{|c|c|c|c|c|c|c|c|c|c|c|c|c|c|c|}
\hline \multirow{4}{*}{$\begin{array}{c}\text { Tre } \\
\text { at } \\
\text { me } \\
\text { nt } \\
\text { T1 }\end{array}$} & \multirow{2}{*}{\multicolumn{2}{|c|}{$\begin{array}{c}\text { Seed Source } \\
\text { (Quality) }\end{array}$}} & \multirow{2}{*}{\multicolumn{2}{|c|}{$\%$ Germination }} & \multirow[t]{3}{*}{ Avg. } & \multicolumn{9}{|c|}{21 Days After Sown Seedlings } \\
\hline & & & & & & \multicolumn{2}{|c|}{$\begin{array}{c}\text { Root } \\
\text { length }(\mathrm{cm})\end{array}$} & \multirow[t]{2}{*}{ Avg. } & \multicolumn{2}{|c|}{$\begin{array}{l}\text { Shoot length } \\
\text { (cm) }\end{array}$} & \multirow[t]{2}{*}{ Avg. } & \multicolumn{2}{|c|}{$\begin{array}{c}\text { Seedling vigor } \\
\text { index }\end{array}$} & \multirow[t]{2}{*}{ Avg. } \\
\hline & \multirow{2}{*}{\multicolumn{2}{|c|}{$\begin{array}{l}\text { Seeds from } \\
\text { healthy plant } \\
\text { panicle }\end{array}$}} & V1 & V2 & & V1 & $\mathrm{V} 2$ & & V1 & V2 & & V1 & V2 & \\
\hline & & & 79.75 & 82.25 & 81.0 & 5.95 & 7.85 & 6.90 & 18.04 & 23.13 & 20.58 & 1913.98 & 2551.59 & 2232.78 \\
\hline $\mathbf{T 2}$ & \multicolumn{2}{|c|}{$\begin{array}{l}\text { Seeds from } \\
\text { diseased plant } \\
\text { panicle }\end{array}$} & 66.5 & 67.25 & 66.87 & 5.40 & 6.89 & 6.14 & 16.34 & 19.70 & 18.02 & 1446.46 & 1785.45 & 1615.95 \\
\hline \multicolumn{3}{|c|}{ Average } & $\mathbf{7 3 . 1 2}$ & 74.75 & 73.93 & 5.67 & 7.37 & 6.52 & 17.19 & 21.41 & 19.3 & 1680.22 & 2168.52 & 1924.37 \\
\hline \multirow{3}{*}{\multicolumn{2}{|c|}{$\mathrm{SE}(\mathbf{m})+$}} & & \multicolumn{2}{|c|}{ Variety (V) } & 1.40 & & 0.05 & \multicolumn{2}{|c|}{ 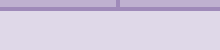 } & 0.40 & \multicolumn{2}{|c|}{ 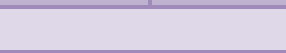 } & 218.13 \\
\hline & & \multicolumn{3}{|c|}{ Seed Source (Quality)(T) } & 1.40 & & & 0.05 & & 0.40 & \multicolumn{2}{|c|}{ 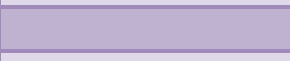 } & 218.13 \\
\hline & & \multicolumn{3}{|c|}{$\mathrm{V} \times \mathrm{T}$} & 1.98 & & & 0.07 & & 0.56 & & & 308.49 \\
\hline \multirow{3}{*}{\multicolumn{2}{|c|}{ CD $(5 \%)$}} & \multicolumn{3}{|c|}{ Variety $(\mathrm{V})$} & 4.49 & & & 0.16 & & 1.27 & \multirow{2}{*}{\multicolumn{2}{|c|}{ 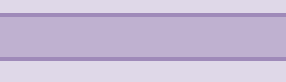 }} & 697.85 \\
\hline & & \multicolumn{3}{|c|}{ Seed Source (Quality)(T) } & 4.49 & & & 0.16 & & 1.27 & & & 697.85 \\
\hline & & \multirow{2}{*}{\multicolumn{3}{|c|}{$\mathrm{V} \times \mathrm{T}$}} & 6.34 & & & 0.22 & & 1.80 & 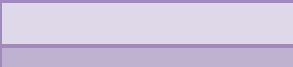 & & 986.91 \\
\hline \multicolumn{2}{|c|}{ CV $(\%)$} & & & & 5.36 & & & 2.11 & & & 5.84 & & & 29.56 \\
\hline
\end{tabular}

V1- Swarna V2- Mahamaya *Average of four replications *Square root transformation

Table.4 Effect of sheath blight incidence on seedling vigor under field conditions (Year-2016)

Design - Factorial RBD; Treatments-4; Replication-4

\begin{tabular}{|c|c|c|c|c|c|c|c|c|c|c|c|c|c|c|}
\hline \multirow{4}{*}{$\begin{array}{c}\text { Tre } \\
\text { at } \\
\text { me } \\
\text { nt } \\
\text { T1 }\end{array}$} & \multirow{2}{*}{\multicolumn{2}{|c|}{$\begin{array}{c}\text { Seed Source } \\
\text { (Quality) }\end{array}$}} & \multirow{2}{*}{\multicolumn{2}{|c|}{$\begin{array}{c}\% \\
\text { Germination }\end{array}$}} & \multirow[t]{3}{*}{ Avg. } & \multicolumn{9}{|c|}{30 Days After Sown Seedlings } \\
\hline & & & & & & \multicolumn{2}{|c|}{$\begin{array}{c}\text { Root } \\
\text { length }(\mathrm{cm})\end{array}$} & \multirow[t]{2}{*}{ Avg. } & \multicolumn{2}{|c|}{$\begin{array}{l}\text { Shoot length } \\
\text { (cm) }\end{array}$} & \multirow[t]{2}{*}{ Avg. } & \multicolumn{2}{|c|}{$\begin{array}{c}\text { Seedling vigor } \\
\text { index }\end{array}$} & \multirow[t]{2}{*}{ Avg. } \\
\hline & \multirow{2}{*}{\multicolumn{2}{|c|}{$\begin{array}{l}\text { Seeds from } \\
\text { healthy plant } \\
\text { panicle }\end{array}$}} & V1 & $\mathrm{V} 2$ & & V1 & V2 & & V1 & V2 & & V1 & V2 & \\
\hline & & & 79.75 & 82.25 & 81.0 & 8.73 & 11.80 & 10.26 & 25.65 & 33.27 & 29.46 & 2747.21 & 3714.33 & 3230.77 \\
\hline $\mathbf{T 2}$ & \multicolumn{2}{|c|}{$\begin{array}{c}\text { Seeds from } \\
\text { diseased plant } \\
\text { panicle }\end{array}$} & 66.5 & 67.25 & 66.87 & 7.57 & 9.95 & 8.76 & 20.77 & 31.56 & 26.16 & 1884.76 & 2787.44 & 2336.10 \\
\hline \multicolumn{3}{|c|}{ Average } & 73.12 & $\mathbf{7 4 . 7 5}$ & 73.93 & 8.15 & 10.87 & 9.51 & 23.21 & 32.41 & 27.81 & 2315.98 & 3250.88 & 2783.43 \\
\hline \multirow{3}{*}{\multicolumn{2}{|c|}{$\mathbf{S E}(\mathbf{m})+$}} & \multirow{2}{*}{\multicolumn{3}{|c|}{$\begin{array}{c}\text { Varıety }(\mathrm{V}) \\
\text { Seed Source (Quality)(T) }\end{array}$}} & 1.40 & & 0.10 & \multicolumn{2}{|c|}{ 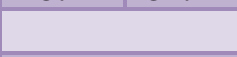 } & 0.32 & \multicolumn{2}{|l|}{2015.00} & 68.50 \\
\hline & & & & & 1.40 & & & 0.10 & & & 0.32 & & & 68.50 \\
\hline & & \multicolumn{3}{|c|}{$\mathrm{V} \times \mathrm{T}$} & 1.98 & & & 0.15 & & 0.45 & \multicolumn{2}{|c|}{ 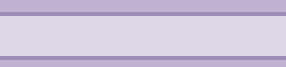 } & 96.88 \\
\hline \multirow{3}{*}{\multicolumn{2}{|c|}{ CD (5\%) }} & \multicolumn{3}{|c|}{ Variety (V) } & 4.49 & & & $\mathbf{0 . 3 3}$ & & & 1.01 & 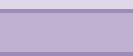 & 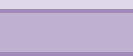 & 219.16 \\
\hline & & \multicolumn{3}{|c|}{ Seed Source (Quality)(T) } & 4.49 & & & $\mathbf{0 . 3 3}$ & & & 1.01 & & & 219.16 \\
\hline & & \multicolumn{3}{|c|}{$\mathrm{V} \times \mathrm{T}$} & 6.34 & & & 0.47 & & & 1.43 & & & 209.93 \\
\hline \multicolumn{2}{|c|}{ CV (\%) } & & & & 5.36 & & & 3.07 & & & 3.21 & & & 6.96 \\
\hline
\end{tabular}

V1- Swarna V2- Mahamaya *Average of four replications 
Table.5 Effect of sheath blight incidence on seedling vigor under Pot conditions (Year-2017) Impact of sheath blight on seedlings and plant vigour

Design - Factorial RBD; Treatments-4; Replication-4

\begin{tabular}{|c|c|c|c|c|c|c|c|c|c|c|c|c|c|c|}
\hline \multirow{4}{*}{$\begin{array}{c}\text { Tre } \\
\text { at } \\
\text { me } \\
\text { nt } \\
\text { T1 }\end{array}$} & \multirow{2}{*}{\multicolumn{2}{|c|}{$\begin{array}{l}\text { Seed Source } \\
\text { (Quality) }\end{array}$}} & \multirow{2}{*}{\multicolumn{2}{|c|}{$\begin{array}{c}\% \\
\text { Germination }\end{array}$}} & \multirow[t]{3}{*}{ Avg. } & \multicolumn{9}{|c|}{21 Days After Sown Seedlings } \\
\hline & & & & & & \multicolumn{2}{|c|}{$\begin{array}{c}\text { Root } \\
\text { length }(\mathrm{cm})\end{array}$} & \multirow[t]{2}{*}{ Avg. } & \multicolumn{2}{|c|}{$\begin{array}{l}\text { Shoot length } \\
(\mathrm{cm})\end{array}$} & \multirow[t]{2}{*}{ Avg. } & \multicolumn{2}{|c|}{$\begin{array}{c}\text { Seedling vigor } \\
\text { index }\end{array}$} & \multirow[t]{2}{*}{ Avg. } \\
\hline & \multirow{2}{*}{\multicolumn{2}{|c|}{$\begin{array}{l}\text { Seeds from } \\
\text { healthy plant } \\
\text { panicle }\end{array}$}} & V1 & V2 & & V1 & V2 & & V1 & V2 & & V1 & V2 & \\
\hline & & & 75.0 & 78.0 & 76.5 & 5.24 & 6.21 & 5.72 & 15.03 & 18.09 & 16.56 & 1520.68 & 1897.68 & 1709.18 \\
\hline T2 & \multicolumn{2}{|c|}{$\begin{array}{l}\text { Seeds from } \\
\text { diseased plant } \\
\text { panicle }\end{array}$} & 60.5 & 65.25 & 62.87 & 4.75 & 5.64 & 5.19 & 13.38 & 14.53 & 13.95 & 1097.79 & 1347.23 & 1222.51 \\
\hline \multicolumn{3}{|c|}{ Average } & 67.75 & 71.62 & 69.68 & 4.99 & 5.92 & 5.45 & 14.20 & 16.31 & 15.25 & 1309.23 & 1622.45 & 1465.84 \\
\hline \multirow{3}{*}{\multicolumn{2}{|c|}{ SE(m)+ }} & \multicolumn{3}{|c|}{ Variety $(\mathrm{V})$} & 0.37 & & 0.10 & & 0.28 & \multicolumn{2}{|c|}{ (2) } & 26.88 \\
\hline & & \multicolumn{3}{|c|}{$\begin{array}{l}\text { Seed Source } \\
\text { (Quality)(T) }\end{array}$} & 0.37 & & 0.10 & & 0.28 & \multirow{2}{*}{\multicolumn{2}{|c|}{ 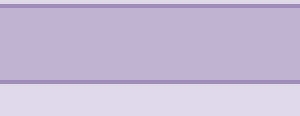 }} & 26.88 \\
\hline & & \multicolumn{3}{|c|}{$\mathrm{V} \times \mathrm{T}$} & 0.53 & & & 0.15 & & & 0.40 & & & 38.01 \\
\hline \multirow{4}{*}{\multicolumn{2}{|c|}{ CD $(5 \%)$}} & \multicolumn{3}{|c|}{ Variety (V) } & 1.20 & & & 0.34 & & 0.40 & & & 85.98 \\
\hline & & \multicolumn{3}{|c|}{$\begin{array}{l}\text { Seed Source } \\
\text { (Quality)(T) }\end{array}$} & 1.20 & & & 0.34 & & 0.40 & & & 85.98 \\
\hline & & \multirow{2}{*}{\multicolumn{3}{|c|}{$\mathrm{V} \times \mathrm{T}$}} & 1.69 & & & 0.47 & & & 0.56 & & & 121.60 \\
\hline & CV (\%) & & & & 1.52 & & & 5.43 & & & 5.14 & & & 5.19 \\
\hline
\end{tabular}

Table.6 Effect of sheath blight incidence on seedling vigor under Pot conditions (Year-2017)

Design - Factorial RBD; Treatments-4; Replication-4

\begin{tabular}{|c|c|c|c|c|c|c|c|c|c|c|c|c|c|c|}
\hline \multirow{4}{*}{ 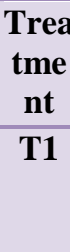 } & \multirow{2}{*}{\multicolumn{2}{|c|}{$\begin{array}{l}\text { Seed Source } \\
\text { (Quality) }\end{array}$}} & \multirow{2}{*}{\multicolumn{2}{|c|}{$\begin{array}{c}\% \\
\text { Germination }\end{array}$}} & \multirow[t]{3}{*}{ Avg. } & \multicolumn{9}{|c|}{30 Days After Sown Seedlings } \\
\hline & & & & & & \multicolumn{2}{|c|}{$\begin{array}{c}\text { Root } \\
\text { length }(\mathrm{cm})\end{array}$} & \multirow[t]{2}{*}{ Avg. } & \multicolumn{2}{|c|}{$\begin{array}{l}\text { Shoot length } \\
\text { (cm) }\end{array}$} & \multirow[t]{2}{*}{ Avg. } & \multicolumn{2}{|c|}{$\begin{array}{c}\text { Seedling vigor } \\
\text { index }\end{array}$} & \multirow[t]{2}{*}{ Avg. } \\
\hline & \multirow{2}{*}{\multicolumn{2}{|c|}{$\begin{array}{l}\text { Seeds from } \\
\text { healthy plant } \\
\text { panicle }\end{array}$}} & V1 & V2 & & V1 & V2 & & $\mathrm{V} 1$ & V2 & & $\mathrm{V} 1$ & V2 & \\
\hline & & & 75.0 & 78.0 & 76.5 & 8.09 & 10.77 & 9.43 & 19.70 & 24.66 & 22.18 & 2087.26 & 2761.67 & 2424.46 \\
\hline $\mathbf{T 2}$ & \multicolumn{2}{|c|}{$\begin{array}{c}\text { Seeds from } \\
\text { diseased plant } \\
\text { panicle }\end{array}$} & 60.5 & 65.25 & 62.87 & 6.76 & 8.57 & 7.66 & 16.44 & 20.17 & 18.30 & 1403.64 & 1876.97 & 1640.30 \\
\hline \multicolumn{3}{|c|}{ Average } & 67.75 & 71.62 & 69.68 & 7.425 & 9.67 & 8.54 & 18.07 & 22.41 & 20.24 & 1745.45 & 2319.32 & 2032.38 \\
\hline \multirow{3}{*}{\multicolumn{2}{|c|}{ SE(m)+ }} & \multicolumn{3}{|c|}{ Variety (V) } & 0.37 & & & 0.25 & & & 0.38 & & & 40.36 \\
\hline & & \multicolumn{3}{|c|}{ Seed Source (Quality)(T) } & 0.37 & & & 0.25 & & & 0.38 & & & 40.36 \\
\hline & & \multicolumn{3}{|c|}{ V x T } & 0.53 & & & 0.35 & & & 0.53 & & & $\mathbf{5 7 . 0 7}$ \\
\hline \multirow{3}{*}{\multicolumn{2}{|c|}{ CD (5\%) }} & \multicolumn{3}{|c|}{ Variety $(\mathrm{V})$} & 1.20 & & & 0.79 & & & 1.20 & & & 129.11 \\
\hline & & \multicolumn{3}{|c|}{ Seed Source (Quality)(T) } & 1.20 & & & 0.79 & & & 1.20 & & & 129.11 \\
\hline & & \multicolumn{3}{|c|}{$\mathrm{V} \times \mathrm{T}$} & 1.69 & & & 1.11 & & & 1.70 & & & 182.59 \\
\hline \multicolumn{2}{|c|}{ CV (\%) } & & & & 1.52 & & & 8.13 & & & 5.25 & & & 5.62 \\
\hline
\end{tabular}

V1- Swarna V2- Mahamaya *Average of four replications 
Table.7 Effect of sheath blight incidence on seedling vigor under field conditions (Year-2017)

\begin{tabular}{|c|c|c|c|c|c|c|c|c|c|c|c|c|c|c|}
\hline \multirow{4}{*}{$\begin{array}{c}\begin{array}{c}\text { Trea } \\
\text { tme } \\
\text { nt }\end{array} \\
\text { T1 }\end{array}$} & \multirow{2}{*}{\multicolumn{2}{|c|}{$\begin{array}{c}\text { Seed Source } \\
\text { (Quality) }\end{array}$}} & \multirow{2}{*}{\multicolumn{2}{|c|}{$\begin{array}{c}\% \\
\text { Germination }\end{array}$}} & \multirow[t]{3}{*}{ Avg. } & \multicolumn{9}{|c|}{21 Days After Sown Seedlings } \\
\hline & & & & & & \multicolumn{2}{|c|}{$\begin{array}{c}\text { Root } \\
\text { length }(\mathrm{cm})\end{array}$} & \multirow[t]{2}{*}{ Avg. } & \multicolumn{2}{|c|}{$\begin{array}{l}\text { Shoot length } \\
\text { (cm) }\end{array}$} & \multirow[t]{2}{*}{ Avg. } & \multicolumn{2}{|c|}{$\begin{array}{c}\text { Seedling vigor } \\
\text { index }\end{array}$} & \multirow[t]{2}{*}{ Avg. } \\
\hline & \multirow{2}{*}{\multicolumn{2}{|c|}{$\begin{array}{l}\text { Seeds from } \\
\text { healthy plant } \\
\text { panicle }\end{array}$}} & V1 & V2 & & V1 & $\mathrm{V} 2$ & & V1 & $\mathrm{V} 2$ & & V1 & V2 & \\
\hline & & & 78.25 & 80.0 & 79.12 & 6.12 & 8.11 & 7.11 & 18.4 & 23.71 & 21.05 & 1920.22 & 2548.08 & 2234.15 \\
\hline $\mathbf{T 2}$ & \multicolumn{2}{|c|}{$\begin{array}{c}\text { Seeds from } \\
\text { diseased plant } \\
\text { panicle }\end{array}$} & 62.75 & 66.75 & 64.75 & 5.35 & 6.92 & 6.13 & 16.24 & 19.59 & 17.91 & 1355.17 & 1772.37 & 1563.77 \\
\hline \multicolumn{3}{|c|}{ Average } & 70.5 & 73.37 & 71.93 & 5.73 & 7.51 & 6.62 & 17.32 & 21.65 & 19.48 & 1637.69 & 2160.22 & 1898.96 \\
\hline \multirow{3}{*}{\multicolumn{2}{|c|}{$\mathrm{SE}(\mathbf{m})+$}} & \multicolumn{3}{|c|}{ Variety $(\mathrm{V})$} & 0.74 & & & 0.08 & & & 0.42 & & & 47.86 \\
\hline & & \multicolumn{3}{|c|}{ Seed Source (Quality)(T) } & 0.74 & & & 0.08 & & & 0.42 & & & 47.86 \\
\hline & & \multicolumn{3}{|c|}{$\mathrm{V} \times \mathrm{T}$} & 1.04 & & & 0.11 & & & 0.59 & & & 67.69 \\
\hline \multirow{3}{*}{\multicolumn{2}{|c|}{ CD $(5 \%)$}} & \multicolumn{3}{|c|}{ Variety (V) } & 2.36 & & & 0.26 & & & 1.34 & & & 153.13 \\
\hline & & \multicolumn{3}{|c|}{ Seed Source (Quality)(T) } & 2.36 & & & 0.26 & & & 1.34 & & & 153.13 \\
\hline & & \multicolumn{3}{|c|}{$\mathrm{V} \times \mathrm{T}$} & 3.33 & & & 0.37 & & & 1.89 & & & 216.55 \\
\hline \multicolumn{2}{|c|}{$\mathrm{CV}(\%)$} & & & & 2.90 & & & 3.45 & & & 6.06 & & & 7.13 \\
\hline
\end{tabular}

Table.8 Effect of sheath blight incidence on seedling vigor under field conditions (Year-2017)

\begin{tabular}{|c|c|c|c|c|c|c|c|c|c|c|c|c|c|c|}
\hline \multirow{4}{*}{$\begin{array}{c}\text { Trea } \\
\text { tme } \\
\text { nt }\end{array}$} & \multirow{2}{*}{\multicolumn{2}{|c|}{$\begin{array}{c}\text { Seed Source } \\
\text { (Quality) }\end{array}$}} & \multirow{2}{*}{\multicolumn{2}{|c|}{$\begin{array}{c}\% \\
\text { Germination }\end{array}$}} & \multirow[t]{3}{*}{ Avg. } & \multicolumn{9}{|c|}{30 Days After Sown Seedlings } \\
\hline & & & & & & \multicolumn{2}{|c|}{$\begin{array}{c}\text { Root } \\
\text { length }(\mathrm{cm})\end{array}$} & \multirow[t]{2}{*}{ Avg. } & \multicolumn{2}{|c|}{$\begin{array}{l}\text { Shoot length } \\
\text { (cm) }\end{array}$} & \multirow[t]{2}{*}{ Avg. } & \multicolumn{2}{|c|}{$\begin{array}{c}\text { Seedling vigor } \\
\text { index }\end{array}$} & \multirow[t]{2}{*}{ Avg. } \\
\hline & \multirow{2}{*}{\multicolumn{2}{|c|}{$\begin{array}{c}\text { Seeds from } \\
\text { healthy plant } \\
\text { panicle }\end{array}$}} & V1 & $\mathrm{V} 2$ & & V1 & V2 & & V1 & V2 & & V1 & V2 & \\
\hline & & & 78.25 & 80.0 & 79.12 & 9.06 & 12.05 & 10.55 & 25.88 & 33.47 & 29.67 & 2442.93 & 3647.95 & 3045.44 \\
\hline T2 & \multicolumn{2}{|c|}{$\begin{array}{c}\text { Seeds from } \\
\text { diseased plant } \\
\text { panicle }\end{array}$} & 62.75 & 66.75 & 64.75 & 7.59 & 9.80 & 8.69 & 20.90 & 31.16 & 26.03 & 1789.52 & 2737.81 & 2263.66 \\
\hline \multicolumn{3}{|c|}{ Average } & 70.5 & 73.37 & 71.93 & 8.32 & 10.92 & 9.62 & 23.39 & 32.31 & 27.85 & 2116.23 & 3192.88 & 2654.55 \\
\hline \multirow{3}{*}{\multicolumn{2}{|c|}{$\mathrm{SE}(\mathbf{m})+$}} & \multicolumn{3}{|c|}{ Variety (V) } & 0.74 & & & 0.15 & & & 0.30 & & & 112.22 \\
\hline & & \multicolumn{3}{|c|}{ Seed Source (Quality)(T) } & 0.74 & & & 0.15 & & & 0.30 & & & 112.22 \\
\hline & & \multicolumn{3}{|c|}{$\mathrm{Vx} \mathrm{T}$} & 1.04 & & & 0.22 & & & 0.43 & & & 158.71 \\
\hline \multirow{3}{*}{\multicolumn{2}{|c|}{ CD (5\%) }} & \multicolumn{3}{|c|}{ Variety $(\mathrm{V})$} & 2.36 & & & 0.49 & & & 0.98 & & & 359.02 \\
\hline & & \multicolumn{3}{|c|}{ Seed Source (Quality)(T) } & 2.36 & & & 0.49 & & & 0.98 & & & 359.02 \\
\hline & & \multicolumn{3}{|c|}{$\mathrm{VxT}$} & 3.33 & & & 0.69 & & & 1.38 & & & 507.73 \\
\hline \multicolumn{2}{|c|}{ CV (\%) } & & & & 2.90 & & & 4.47 & & & 3.09 & & & 11.96 \\
\hline
\end{tabular}

V1- Swarna V2- Mahamaya *Average of four replications * Square root transformation 
Table.9 Effect of sheath blight incidence on seedling vigor under Pot conditions (Pooled) Impact of sheath blight on seedlings and plant vigour

Design - Factorial RBD; Treatments-4; Replication-4

\begin{tabular}{|c|c|c|c|c|c|c|c|c|c|c|c|c|c|c|}
\hline \multirow{4}{*}{$\begin{array}{c}\text { Tre } \\
\text { at } \\
\text { me } \\
\text { nt } \\
\text { T1 }\end{array}$} & \multirow{2}{*}{\multicolumn{2}{|c|}{$\begin{array}{c}\text { Seed } \\
\text { Source } \\
\text { (Quality) }\end{array}$}} & \multirow{2}{*}{\multicolumn{2}{|c|}{$\begin{array}{c}\% \\
\text { Germinati } \\
\text { on }\end{array}$}} & \multirow[t]{3}{*}{ Avg. } & \multicolumn{9}{|c|}{21 Days After Sown Seedlings } \\
\hline & & & & & & \multicolumn{2}{|c|}{$\begin{array}{c}\text { Root } \\
\text { length }(\mathrm{cm})\end{array}$} & \multirow[t]{2}{*}{ Avg. } & \multicolumn{2}{|c|}{$\begin{array}{l}\text { Shoot length } \\
\quad(\mathbf{c m})\end{array}$} & \multirow[t]{2}{*}{ Avg. } & \multicolumn{2}{|c|}{$\begin{array}{l}\text { Seedling vigor } \\
\text { index }\end{array}$} & \multirow[t]{2}{*}{ Avg. } \\
\hline & \multirow{2}{*}{\multicolumn{2}{|c|}{$\begin{array}{l}\text { Seeds from } \\
\text { healthy } \\
\text { plant } \\
\text { panicle }\end{array}$}} & V1 & $\mathrm{V} 2$ & & V1 & V2 & & V1 & V2 & & V1 & V2 & \\
\hline & & & $\begin{array}{c}76 . \\
0\end{array}$ & $\begin{array}{c}79.2 \\
5\end{array}$ & 77.62 & 5.14 & 6.08 & 5.60 & 15.32 & 18.1 & 16.71 & 1555.75 & 1917.25 & 1736.5 \\
\hline $\mathbf{T 2}$ & \multicolumn{2}{|c|}{$\begin{array}{l}\text { Seeds from } \\
\text { diseased } \\
\text { plant } \\
\text { panicle }\end{array}$} & $\begin{array}{c}62 . \\
0\end{array}$ & 66.0 & 63.99 & 4.71 & 5.57 & $\begin{array}{c}5.1 \\
3\end{array}$ & 13.23 & 14.48 & 13.85 & 1113.85 & 1340.81 & $\begin{array}{c}1227.3 \\
3\end{array}$ \\
\hline \multicolumn{3}{|c|}{ Average } & 69. & 79.0 & 80.5 & 79.75 & 5.23 & 6.40 & 5.82 & 15.41 & 19.22 & 17.31 & 1630.92 & 2063.21 \\
\hline \multirow{3}{*}{\multicolumn{2}{|c|}{$\mathrm{SE}(\mathbf{m})+$}} & \multicolumn{3}{|c|}{ Variety (V) } & 0.90 & & & 0.10 & & & 0.33 & & & 35.71 \\
\hline & & \multicolumn{3}{|c|}{$\begin{array}{l}\text { Seed Source } \\
\text { (Quality)(T) }\end{array}$} & 0.90 & & 0.10 & & 0.33 & & & 35.71 \\
\hline & & \multicolumn{3}{|c|}{$\mathrm{V} \times \mathrm{T}$} & 1.27 & & & 0.14 & & & 0.47 & & & $\mathbf{5 0 . 5 0}$ \\
\hline \multirow{3}{*}{\multicolumn{2}{|c|}{ CD (5\%) }} & \multicolumn{3}{|c|}{ Variety (V) } & 2.88 & & & 0.33 & & & 1.06 & & & 114.23 \\
\hline & & \multicolumn{3}{|c|}{$\begin{array}{l}\text { Seed Source } \\
\text { (Quality)(T) }\end{array}$} & 2.88 & & & 0.33 & & & 1.06 & & & 114.23 \\
\hline & & \multicolumn{3}{|c|}{$\mathrm{V} \times \mathrm{T}$} & 4.08 & & & 0.46 & & & 1.50 & & & 161.55 \\
\hline \multicolumn{2}{|c|}{ CV (\%) } & & & & 3.60 & & & 5.37 & & & 6.13 & & & 6.82 \\
\hline
\end{tabular}

V1- Swarna V2- Mahamaya *Average of four replications * Square root transformation

Table.10 Effect of sheath blight incidence on seedling vigor under Pot conditions (Pooled)

\begin{tabular}{|c|c|c|c|c|c|c|c|c|c|c|c|c|c|c|}
\hline \multirow{4}{*}{$\begin{array}{c}\text { Tre } \\
\text { at } \\
\text { me } \\
\text { nt } \\
\text { T1 }\end{array}$} & \multirow{2}{*}{\multicolumn{2}{|c|}{$\begin{array}{l}\text { Seed Source } \\
\text { (Quality) }\end{array}$}} & \multirow{2}{*}{\multicolumn{2}{|c|}{ \% Germination }} & \multirow[t]{3}{*}{ Avg. } & \multicolumn{9}{|c|}{30 Days After Sown Seedlings } \\
\hline & & & & & & \multicolumn{2}{|c|}{$\begin{array}{c}\text { Root } \\
\text { length }(\mathbf{c m})\end{array}$} & \multirow[t]{2}{*}{ Avg. } & \multicolumn{2}{|c|}{$\begin{array}{l}\text { Shoot length } \\
\text { (cm) }\end{array}$} & \multirow[t]{2}{*}{ Avg. } & \multicolumn{2}{|c|}{$\begin{array}{c}\text { Seedling vigor } \\
\text { index }\end{array}$} & \multirow[t]{2}{*}{ Avg. } \\
\hline & \multirow{2}{*}{\multicolumn{2}{|c|}{$\begin{array}{l}\text { Seeds from } \\
\text { healthy plant } \\
\text { panicle }\end{array}$}} & V1 & V2 & & V1 & V2 & & V1 & V2 & & V1 & V2 & \\
\hline & & & 76 & 79.25 & 77.625 & 8.11 & 10.825 & 9.465 & 19.58 & 24.735 & 22.155 & 2108.155 & 2816.25 & 2462.2 \\
\hline T2 & \multicolumn{2}{|c|}{$\begin{array}{l}\text { Seeds from } \\
\text { diseased plant } \\
\text { panicle }\end{array}$} & 62 & 66 & 63.995 & 6.765 & 8.595 & 7.675 & 16.57 & 20.2 & 18.38 & 1446.675 & 1899.43 & 1673.05 \\
\hline \multicolumn{3}{|c|}{ Average } & 72.62 & 70.81 & 7.43 & 9.71 & 8.57 & $\begin{array}{c}18.0 \\
7\end{array}$ & 22.46 & 20.26 & $\begin{array}{c}1777 . \\
41\end{array}$ & 2357.84 & 2067.62 & 72.62 \\
\hline \multirow{3}{*}{\multicolumn{2}{|c|}{$\mathbf{S E}(\mathbf{m})+$}} & \multicolumn{3}{|c|}{ Variety $(\mathrm{V})$} & 0.90 & & & 0.25 & & & 0.37 & & & 44.66 \\
\hline & & \multicolumn{3}{|c|}{ Seed Source (Quality)(T) } & 0.90 & & & 0.25 & & & 0.37 & & & 44.66 \\
\hline & & \multicolumn{3}{|c|}{ V x T } & 1.27 & & & 0.35 & & & 0.52 & & & 63.16 \\
\hline \multirow{3}{*}{\multicolumn{2}{|c|}{ CD (5\%) }} & \multicolumn{3}{|c|}{ Variety (V) } & 2.88 & & & 0.35 & & & 1.17 & & & 142.87 \\
\hline & & \multicolumn{3}{|c|}{ Seed Source (Quality)(T) } & 2.88 & & & 0.35 & & & 1.17 & & & 142.87 \\
\hline & & \multicolumn{3}{|c|}{$\mathrm{Vx} \mathrm{T}$} & 4.08 & & & 0.50 & & & 1.65 & & & 202.05 \\
\hline \multicolumn{2}{|c|}{ CV (\%) } & & & & 3.60 & & & 8.22 & & & 5.10 & & & 6.11 \\
\hline
\end{tabular}


Table.11 Effect of sheath blight incidence on seedling vigor under field conditions (Pooled)

Design - Factorial RBD; Treatments-4; Replication-4

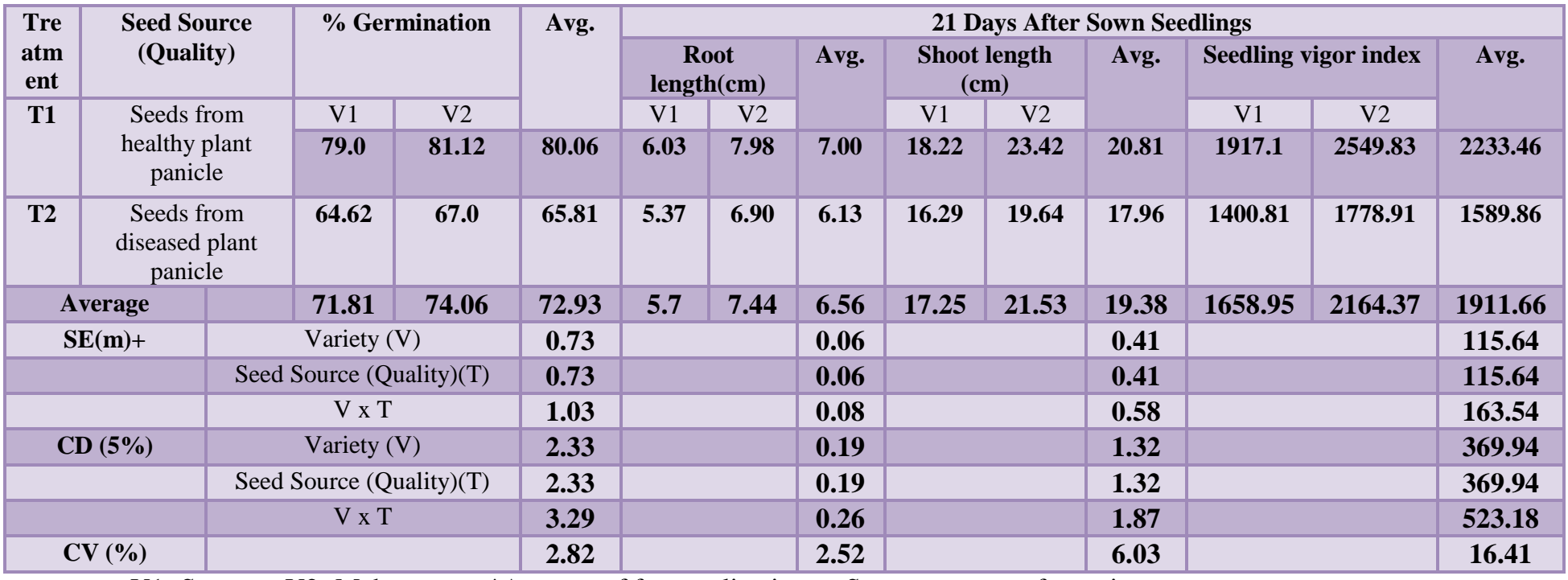

V1- Swarna V2- Mahamaya *Average of four replications Square root transformation

Table.12 Effect of sheath blight incidence on seedling vigor under field conditions (Pooled)

\begin{tabular}{|c|c|c|c|c|c|c|c|c|c|c|c|c|c|c|}
\hline \multirow{4}{*}{$\begin{array}{c}\text { Tre } \\
\text { at } \\
\text { me } \\
\text { nt } \\
\text { T1 }\end{array}$} & \multirow{2}{*}{\multicolumn{2}{|c|}{$\begin{array}{l}\text { Seed Source } \\
\text { (Quality) }\end{array}$}} & \multirow{2}{*}{\multicolumn{2}{|c|}{$\begin{array}{c}\% \\
\text { Germination }\end{array}$}} & \multirow[t]{3}{*}{ Avg. } & \multicolumn{9}{|c|}{30 Days After Sown Seedlings } \\
\hline & & & & & & \multicolumn{2}{|c|}{$\begin{array}{c}\text { Root } \\
\text { length }(\mathrm{cm})\end{array}$} & \multirow[t]{2}{*}{ Avg. } & \multicolumn{2}{|c|}{$\begin{array}{l}\text { Shoot length } \\
(\mathrm{cm})\end{array}$} & \multirow[t]{2}{*}{ Avg. } & \multicolumn{2}{|c|}{$\begin{array}{c}\text { Seedling vigor } \\
\text { index }\end{array}$} & \multirow[t]{2}{*}{ Avg. } \\
\hline & \multirow{2}{*}{\multicolumn{2}{|c|}{$\begin{array}{l}\text { Seeds from } \\
\text { healthy plant } \\
\text { panicle }\end{array}$}} & V1 & V2 & & V1 & V2 & & V1 & V2 & & V1 & V2 & \\
\hline & & & 79.0 & 81.12 & 80.06 & 8.89 & 11.92 & 10.40 & 25.76 & 33.37 & 29.56 & 2595.07 & 3681.14 & 3138.10 \\
\hline $\mathbf{T 2}$ & \multicolumn{2}{|c|}{$\begin{array}{l}\text { Seeds from } \\
\text { diseased plant } \\
\text { panicle }\end{array}$} & 64.62 & 67.0 & 65.81 & 7.58 & 9.87 & 8.72 & 20.83 & 31.36 & 26.09 & 1837.14 & 2762.62 & 2299.88 \\
\hline \multicolumn{3}{|c|}{ Average } & 71.81 & 74.06 & 72.93 & 8.23 & 10.89 & 9.56 & 23.29 & 32.36 & 27.82 & 2216.10 & 3221.88 & 2718.99 \\
\hline \multirow{3}{*}{\multicolumn{2}{|c|}{$\mathrm{SE}(\mathbf{m})+$}} & \multicolumn{3}{|c|}{ Variety $(\mathrm{V})$} & 0.73 & & & 0.12 & & & $\mathbf{0 . 3 1}$ & & & $\mathbf{7 2 . 4 0}$ \\
\hline & & \multicolumn{3}{|c|}{ Seed Source (Quality)(T) } & 0.73 & & & 0.12 & & & 0.31 & & & 72.40 \\
\hline & & \multicolumn{3}{|c|}{$\mathrm{V} \times \mathrm{T}$} & 1.03 & & & 0.17 & & & 0.44 & & & 102.39 \\
\hline \multirow{3}{*}{\multicolumn{2}{|c|}{ CD (5\%) }} & \multicolumn{3}{|c|}{ Variety $(\mathrm{V})$} & 2.33 & & & 0.38 & & & 0.99 & & & 231.62 \\
\hline & & \multicolumn{3}{|c|}{ Seed Source (Quality)(T) } & 2.33 & & & 0.38 & & & 0.99 & & & 231.62 \\
\hline & & \multicolumn{3}{|c|}{$\mathrm{V} \times \mathrm{T}$} & 3.29 & & & 0.54 & & & 1.40 & & & 327.56 \\
\hline \multicolumn{2}{|c|}{ CV (\%) } & & & & 2.82 & & & 3.54 & & & 3.15 & & & 7.53 \\
\hline
\end{tabular}

V1- Swarna V2- Mahamaya *Average of four replications Square root transformation 
Fig.1

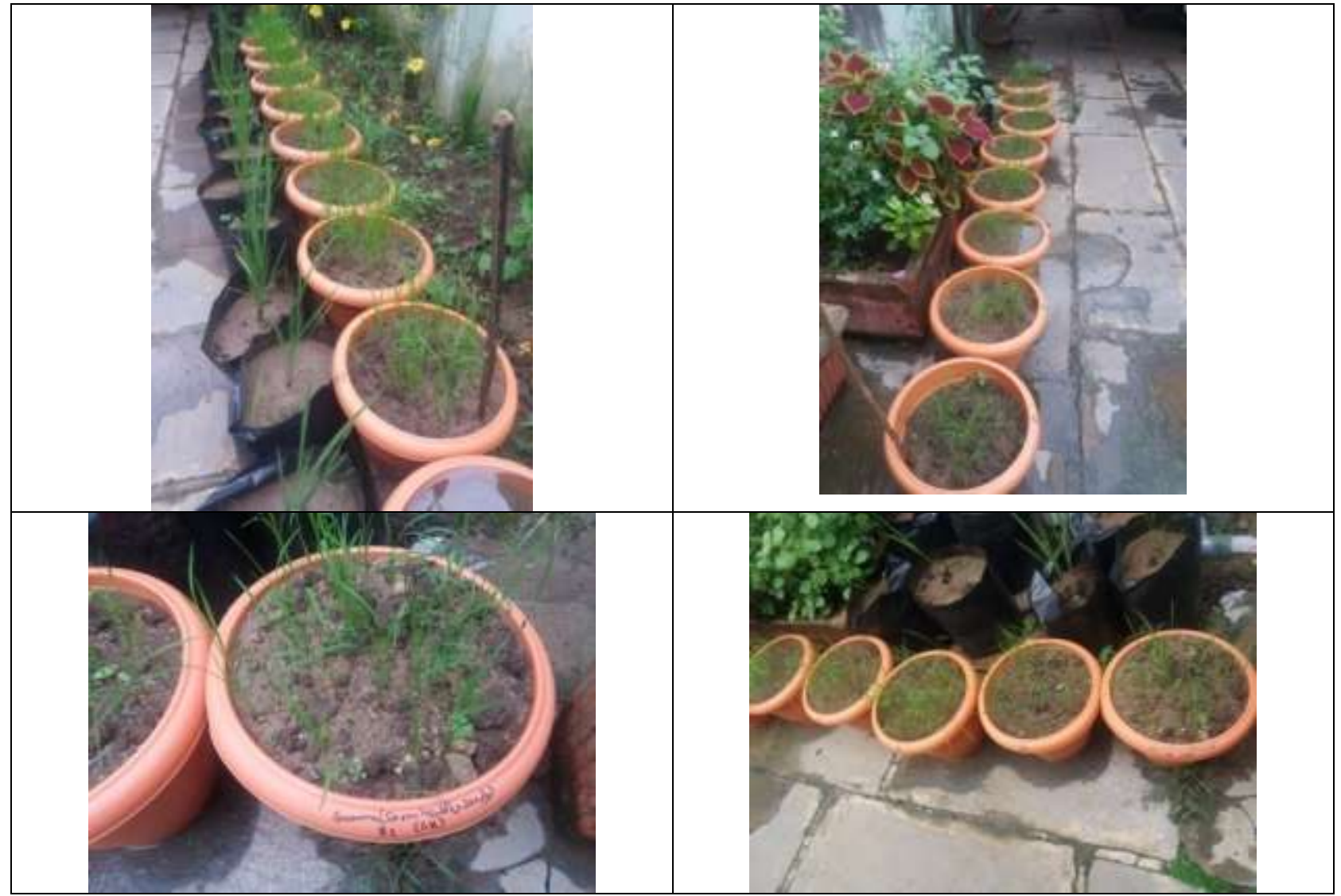

The seedling vigor index during the same period they were statistically significance between healthy and diseased plant seeds in both varieties after 21 days of sowings. The seedling vigour index calculated clearly revealed that healthy plant seeds in both the varieties i.e., Swarna (1917.10) and Mahamaya (2549.83) was more than the corresponding diseased plant seeds (i.e., 1400.81 and 1778.91, respectively). Similar trend was observed in root length of the healthy plant seed of Swarna (8.89) and Mahamaya (11.92) after 30 DAS. The diseased plant seed during the same period recorded root length of 7.58 and 9.87, respectively. The shoot length for the healthy plant seed for Swarna was 25.76 and 33.37 for Mahamaya. The shoot length was also affected in diseased plants seeds which were 20.83 and 31.36 , respectively during the same period of observation. The seedling vigour index calculated clearly revealed that healthy plant seeds in both the varieties i.e., Swarna (2595.07) and Mahamaya (3681.14) was more than the corresponding diseased plant seeds (i.e., 1837.14 and 2762.62, respectively). The statistical analysis also clearly revealed that root, shoot and seedling vigor index differences were significant between the healthy plant seeds (T1) to that of diseased plant seeds (T2).

Seed vigour was recognized by earlier researchers as an important quality parameter and distinct from germinability. Decline in germinability was also attributed to decline in vigour and might be due to several factors including the seed infection with microorganisms (Seshu et al., 1987). Seedlings emerged from seeds of infected panicles had 
poor seedling vigour and reduced root length was more pronounced than reduced shoot length (Hegde and Anahosur, 2000). Most seedlings from infected, partially developed seeds died before reaching maturity.

Also, Velazhahan, (1991) found that the culture filtrates of the rice sheath rot pathogen had a pronounced inhibitory effect on germination in all 7 cultivars tested and also reduced shoot and root lengths of the seedlings. Tripathi and Jain (2005) reported that infection / seed discoloration is becoming a problem in rice growing areas of Madhya Pradesh, India. The seeds are infected with pathogenic fungi under high humidity during flowering and post-flowering stages, which may adversely affect the seed germination and seedling vigour.

\section{Field condition}

During the year 2016 the data in table 1 shows that The highest percent germination under field conditions was obtained in Mahamaya where the healthy seeds (T1) (86.6) and in Swarna (83.00), under field conditions also the seeds from diseased panicles showed reduction in percent germination for Swarna (66.4) or Mahamaya (64.0)

The seeds form healthy plant panicle showed higher percent germination in pot and field conditions as compared to the disease plant panicle seeds.

The root length, shoot length as well as seedling vigor index showed statistically significant longer root length, shoot length and seedling vigor index in both varieties (Swarna and Mahamaya) seed tested from healthy plant panicles in comparison to sheath blight affected plant panicle seeds. Percent seed germination and seedling vigor calculation clearly indicated that healthy plant seed were superior percent germination and seedling vigor.

\section{References}

Acharya, S. and Gupta, P.K.S. (1996). Seedborne nature and seed transmission of rice sheath blight. International Rice Res. Notes. 21: 76-77.

Anselme, C. 1987. Pre-harvest and Postharvest treatment to improve rice seed health. Proc. Int. Workshop on Rice Seed Health, IRRI. 16-20 Mar, pp. 225-234.

Binesh, H. and Torabi, M. 1985. Mode of transmission of rice sheath blight through seeds and reaction of rice cultivars to the disease. Iranian Journal of Plant Pathology. 21: 1-4, 3-5, 15-25.

Dath, S. R. 1990. Sheath blight disease of rice and its management. Associate Publishing Company, New Delhi. 152 pp.

Fakir, G. A., Hossain, I., Ahmed, M. U. Asadud-Doullah, M. and Alam, M. 2002. Quality of farmers Boro and T. Aman rice seeds collected before sowing from Bogra, Rajshahi and Rangpur districts of Bangladesh. A paper presented in the review and planning meeting of the Rice Seed Health Improvement (SHIP), PETRRA project held on 17-18 April at BRRI, Gazipur, Bangladesh.

germination and seedling vigour. Jawaharlal Nehru Krishi Vishwa Vidyalaya (JNKVV) Research-Journal. 39(2): 116-118.

Hegde, Y. R. and Anahosur, K. H. 2000. Effect of false smut of rice on yield components and growth parameters. Indian-Phytopathology.53(2):181-184.

Nanda, J. S. and Chaudhary R. C. 1972. Effect of grain spotting in an early variety of rice 'Padma'. Seedling characteristics. Riso. 21: 1, 7-10.

Neergaard, P. 1979. Seed Pathology. 2nd Vol. The Mac Millan Press Ltd., London and Basingstoks, p.1187. 
Ou, S. H. 1985. Rice diseases. Common Wealth Mycological institute, Kew, England. 2nd ed. 379 pp.

Roy, A.K. (1989). Source of seed-borne infection of sheath blight of rice. Oryza. 26: 111-112.

Seshu, D.V., Krishnasamy, V. and Siddiqui, S. B. 1987. Seed Vigour in rice. Proc. Int. Workshop on Rice Seed Health, IRRI. 16-20 Mar., pp.315-330.
Silvalingam P.N., Vishwakarma S.N. and Singh U.S. (2006) Role of seed-borne inoculum of Rhizoctonia solani in sheath blight of rice. Indian Phytopath. 59 (4) : 445-452.

Tripathi, S. K. and Jain, A. K. 2005. Seed mycoflora of rice and their influence on

\section{How to cite this article:}

Nitin Kumar Toorray, P. K. Tiwari, A. S. Kotasthane and Awadhiya, G. K. 2019. Impact of Sheath Blight Disease of Paddy on Seedlings and Plant Vigor. Int.J.Curr.Microbiol.App.Sci. 8(10): 2460-2473. doi: https://doi.org/10.20546/ijcmas.2019.810.286 